density at the edges is higher than at other places. Considering the end cathode in an electrolytic cell, one can easily see that the extra current lines necessary for getting enhanced current density at the edges can pass not only from the anode to the cathode edge from the front, but also some of the current can pass through the cathode sheet on to the back side and from there to the electrolyte and go on to the edge through the electrolyte. The result is that anodic current would exist at the back side of the end cathode from the points away from the edges to the edges and presence of local anodic and cathodic areas in a plate normally leads to corrosion. Fig. 1 and 2 indicate qualitatively the reversal of current at the edges. Absence of a reversal point at one of the ends in fig. 2 may be due to difficulties inherent in the set up such as gas evolution and orientation of electrodes. This explanation has been confirmed by a crucial experiment that was tried. It was found that the shielding of the edges by making use of glass plate blinkers or shields placed in the gaps between the cathode edges and the sides of the cell, effectively brought down the reversal of the diretion of the current.

(Received Apr. 6, 1961)

\title{
Literature:
}

1) Indra Sanghi, Ramanathan VR, Curr. Sci. 28, 23 (1959).

2) R. S. Thornhill, U. R. Evans, J. Chem. Soc. 2109 (1938).

3) U. R. Evans, "Metallic Corrosion, Passivity and Protection" p. 8 (1948), Edward Arnold (Publishers) Ltd., London.

\section{Regularities and One-parameter Equation for Activity Coefficients of Strong Electrolytic Solutions}

\author{
Makoto MORIYAMA \\ The EIectrical Communication Laboratory, Nippon Telegraph and Telephone Public \\ Corporation, Musashino-shi, Tokyo
}

Using the regularities in the activity coefficients of electrolytic solutions, Glueckauf's two-parameter equation for the stoichiometric mean ionic molal activity coefficient $\gamma_{ \pm}$, based on mole-fractional statistics, can be reduced to a one-parameter equation

$$
\ln \gamma_{ \pm}=-\frac{A \sqrt{m}}{1+B \sqrt{ } m}+\ln \left\{1+\left(\frac{4 A B}{125}\right) m+\frac{7}{8}\left(\frac{4 A B}{125}\right)^{2} m^{2}+\frac{17}{24}\left(\frac{4 A B}{125}\right)^{3} m^{3}\right\}
$$

where $m$ is the concentration in molality, $A$ and $B$ depend on the type of electrolyte and further $B$ is a function of $\alpha$, the mean distance of closest approach. This equation is compared with experimental values. The constancy of $R \gamma$, concentration ratio, is reexamined. Finally Glueckauf's three-parameter equation is discussed in connection with the regularities in the activity coefficient.

\section{Introduction}

In the preceding paper ${ }^{1)}$ we have pointed out that the existence of $\gamma_{ \pm \min }-\sqrt{m_{\min }}$ curve for a series of electrolytes will mean that $\gamma_{ \pm \min }$ may be expressible as a function of $\sqrt{m_{\min }}$ alone. With the use of Glueckauf's equation for the concentration dependence of activity coefficients based on the mole-fractional statistics ${ }^{2}$, the ratio $R \gamma$ of the higher concentration $m_{1}$, at which $\gamma_{ \pm}=1$, to the concentration $m_{\min }$, at 
which $\gamma_{ \pm}=\gamma_{ \pm} \min$, has been expressed approximately ${ }^{3)}$ by

where

$$
R \gamma \equiv m_{1} / m_{\min } \cong(8 / 3)+t^{1 / 3}
$$

$$
t \equiv 4 A B /[\beta(2 h-\nu)]
$$

and

$A=2.303 \cdot 0.509\left|z_{1} z_{2}\right| \sqrt{I / C}$

$B=B(\alpha)=0.329 \alpha \sqrt{I / C}$

$\nu$ : number of ions into which an electrolyte ionizes

$\beta=0.018=M / 1000(M:$ molecular weight of water)

$m$ : molality (moles per $1000 \mathrm{~g}$ solvent)

$\sqrt{I / C}$ : factor depending on the type of electrolyte

$h$ : number of hydrated water molecules per electrolyte molecule

In this paper, with use of Eq. (1), we shall indicate, in the first place, that Glueckauf's equation involving two parameters $\alpha$ and $h^{2) *}$

$$
\ln \gamma_{ \pm}=\frac{A \sqrt{m}}{1+B \sqrt{m}}-\frac{h}{\nu} \ln (1-\beta h m)+\frac{h-\nu}{\nu} \ln [1-\beta(h-\nu) m]
$$

may be reduced to an equation containing only one parameter $B(\alpha)$ or $\alpha$, and then compare the new equation with experimental results. The constancy of $R \gamma$ is reexamined in detail. Finally Glueckauf's threeparameter equation based on the volume fractional statistics is discussed briefly in connection with the regularities in the activity coefficients.

\section{Derivation of One-parameter Equation}

Eq. (3) can be rewritten in the following forms with allowable approximations:

$$
\begin{aligned}
\ln \gamma_{ \pm} & =-\frac{A \sqrt{m}}{1+B \sqrt{m}}+\ln (1-\beta h m)-h / \nu[1-\beta(h-\nu) \mathrm{m}]^{h-\nu / \nu} \\
& \cong-\frac{A \sqrt{m}}{1+B \sqrt{m}}+\ln \left\{1+\beta(2 h-\nu) m+\frac{7}{8} \beta^{2}\left(4 h^{2}-4 h \nu+\frac{8}{7} \nu^{2}\right) m^{2}+\frac{17}{24} \beta^{3}\left(8 h^{3}-12 h^{2} \nu+\frac{116}{17} h \nu^{2}-\frac{24}{17} \nu^{3}\right) m^{3}\right\} \\
& \cong-\frac{A \sqrt{m}}{1+B \sqrt{m}}+\ln \left\{1+\beta(2 h-\nu) m+\frac{7}{8}[\beta(2 h-\nu)]^{2} m^{2}+\frac{17}{24}[\beta(2 h-\nu)]^{3} m^{3}\right\}
\end{aligned}
$$

Or

$$
\ln \gamma_{ \pm} \cong-\frac{A \sqrt{m}}{1+B \sqrt{m}}+\beta(2 h-\nu) m+\frac{3}{8}[\beta(2 h-\nu)]^{2} m^{2}
$$

Inserting the relation $\beta(2 h-\nu)=4 A B / t$ from Eq. (2) into (4), we obtain

$$
\ln \gamma_{ \pm}=-\frac{A \sqrt{m}}{1+B \sqrt{m}}+\ln \left\{1+\left(\frac{4 A B}{t}\right) m+\left(\frac{7}{8}\right)\left(\frac{4 A B}{t}\right)^{2} m^{2}+\left(\frac{17}{24}\right)\left(\frac{4 A B}{t}\right)^{3} m^{3}\right\}
$$

If we put $\overline{R \gamma}$ equal to a mean value $\overline{R \gamma}=7.666^{* *}$, we find the corresponding mean value $\bar{t}=125$ from the relation (1). Substituting $\bar{t}$ into (5), we have an approximate equation

$$
\ln \gamma_{ \pm}=-\frac{A \sqrt{m}}{1+B \sqrt{m}}+\left\{1+\left(\frac{4 A B}{125}\right) m+\frac{7}{8}\left(\frac{4 A B}{125}\right)^{2} m^{2}+\frac{17}{24}\left(\frac{4 A B}{125}\right)^{3} m^{3}\right\}
$$

which contains one parameter $B(\alpha)$ only. Thus the two-parameter equation (3) has been reduced to oneparameter one (6) using the relation (1).

\section{Comparison with Experiments}

The behaviours of Eq. (6) for four types of electrolyte are compared with experimental data ${ }^{4)}$ as shown

* On the right of Eq. (3), a term, which was pointed out by R. H. Stokes and R. A. Robinson [ref.2], has been omitted, because that is negligible compared with the first term. The first term, representing the Debye-Hückel term, has been expressed here approximately in molality $\mathrm{m}$ in place of ionic strength 1 . The other two terms indicate the contribution from the hydrated water and the difference due to the conversion of the molal activity coefficient into the rational one.

** In the preceding paper [ref. 3] we have taken $\overline{R \gamma}=7.666$ so as to obtain the best agreement of the equation for $\gamma_{ \pm m i n}-\sqrt{m_{m i n}}$ with its experimental curve. 
in Figs. 1-4. In the cases of 1-1 and 2-1 types, it seems necessary to assign, in general, somewhat large values to $\alpha$ in order to get better agreement with experimental data. However, the largest value, say $\alpha=7$, gives the poorest agreement. This indicates that $4 A B / 125$ is a little small, that is, $\bar{t}$ is a little large. This tendency will be due to the neglection of the higher order terms in the derivation of $R \gamma^{3)}$. However, taking account of the use of the same mean ratio $\overline{R \gamma}=7.666$ for all types of electrolyte, the agreement must be said quite satisfactory, and which may confirm not only the possibility of one-parameter equation for $\gamma_{ \pm}$ but also the existence of regularities in the activity coefficients.

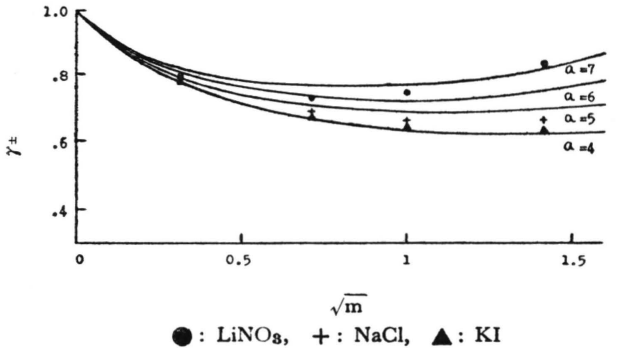

Fig. 1 Plot of $\gamma_{ \pm}$according to Eq. (6) for type $1-1$ at $25^{\circ} \mathrm{C}$

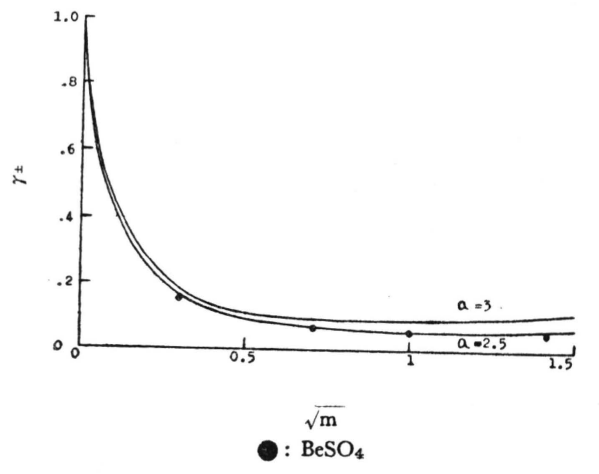

Fig. 3 Plot of $\gamma_{ \pm}$according to Eq. (6) for type $2-2$ at $25^{\circ} \mathrm{C}$

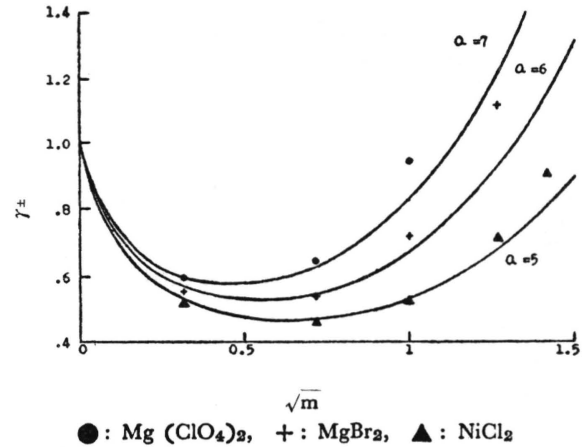

Fig. 2 Plot of $\gamma_{ \pm}$according to Eq. (6) for type $2-1$ at $25^{\circ} \mathrm{C}$

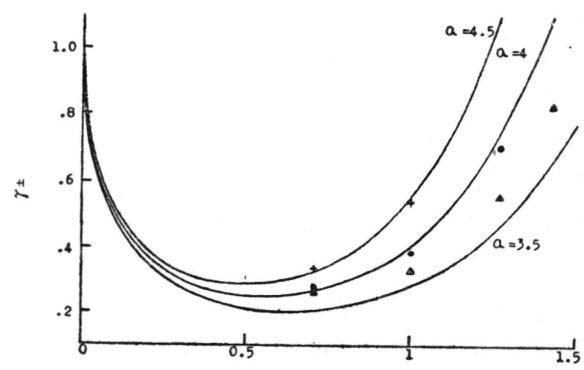

$$
+: \mathrm{AlCl}_{3}, \quad \stackrel{\sqrt{\mathrm{m}}}{:} \stackrel{\mathrm{YCl}}{3}, \Delta: \mathrm{PrCl}_{3}
$$

Fig. 4 Plot of $\gamma_{ \pm}$according to Eq. (6) for type $3-1$ at $25^{\circ} \mathrm{C}$

\section{Discussion}

In the preceding paper ${ }^{3)}$, starting from Glueckauf's equation for $\gamma_{ \pm}$based on the mole fractional statistics (3), we have derived the ratio $R \gamma$ approximately as

$$
\begin{aligned}
R \gamma \equiv m_{1} / m_{\mathrm{min}} & \cong\left(\frac{t^{1 / 2}-1}{t^{1 / 3}-(4 / 3)}\right)^{2} \\
& \cong t^{1 / 3}+(8 / 3)
\end{aligned}
$$

Furthermore, we have assigned to $R \gamma$ a constant value which give the best agreement between experimental data and the equation for $\gamma_{ \pm \min }-\sqrt{m_{\min }}$ curve. In other words, we have assumed to regard $R \gamma$ as constant. Here we shall examine the constancy of $\overline{R \gamma}$ more directly.

If we plot $R \gamma$ against $t$ according to Eq. (7), we obtain a curve illustrated in Fig. 5. Although $R \gamma$ is a complicated function of $t$ as shown in Eq. (7), the curve given in Fig. 5 shows that $\overline{R r}$ may be expressed approximately, over the whole range of $t$ in question, as follows :

$$
R \gamma=6.2+0.0112 t
$$

In view of the fact that $t$ changes in a limited range, we can expect that $R \gamma$ takes also a value in a very limited range. In fact it will be found from Fig. 5 that $R \gamma$ takes the values in a very limited range, that 


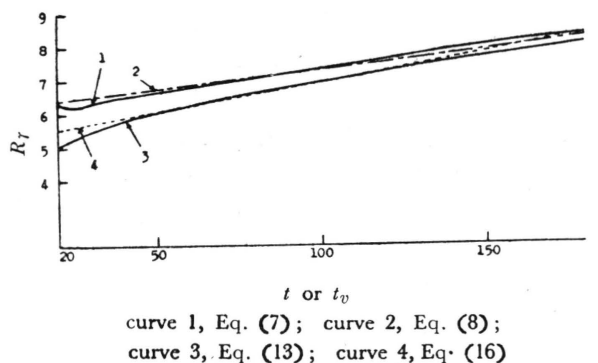

Table 1

\begin{tabular}{c|c|l|l}
\hline $\begin{array}{c}\text { Type of } \\
\text { electrolyte }\end{array}$ & $\left|z_{1} z_{2}\right|$ & $\mathrm{q}$ & \multicolumn{1}{|c}{$E / E^{\prime}$} \\
\hline $1-1$ & 1 & 1 & $D / b \mathbf{3} / \mathbf{2} D$ \\
$2-1,1-2$ & 2 & 2.45 & $D / 0.82 b \mathbf{3} / \mathbf{2} D$ \\
$2-2$ & 4 & 4 & $D / b \mathbf{3} / \mathbf{2} D$ \\
$3-1$ & 3 & 4.24 & $D / 0.71 h \mathbf{3} / \mathbf{2} D$ \\
\hline
\end{tabular}

Fig. 5 Concentration ratio $R \gamma$ as a

function of $t$ or $t_{v}$

is, 6 9. If we make $\overrightarrow{R \gamma}$ equal to a mean value $R_{\gamma}$, the corresponding $\vec{t}$, a mean value of $t$, is obtained, that is

$$
\overline{R \gamma}=6.2+0.0112 \bar{t}
$$

[Putting $\overline{R \gamma}=7.666$ we have $\bar{t}=130$ from Eq. (8) and $\bar{t}=125$ from Eq. (1), therefore both are nearly equal.] Next we shall consider the physical meaning of the constancy of $\bar{E}$.

As already stated ${ }^{3)}, t$ has been written in the form

$$
t=G\left(\frac{q e^{2}}{b^{3 / 2} D \alpha}\right)^{2}=G E^{2}
$$

where

$$
E=c e^{2} / b^{3 / 2} D \alpha
$$

$e$ is the electronic charge, $G=40 \pi N / 3(k T)^{2}, \quad 1>b>0^{*}, q^{2}=\left|z_{1} z_{2}\right|(I / c) . \quad E$ may be regarded as a kind of potential energy between the two ions bearing charges $\left(q^{1 / 2} / b^{3 / 4}\right) e$ which are separated by a distance $\alpha$ in a medium of dielectric constant $D$. From Eqs. (1) and (10) we have

$$
R \gamma-(8 / 3)=t^{1 / 3} \propto E^{2 / 3} \text {. }
$$

In the approximations (1) or (7), therefore, it may not be inadequate to regard $E$, a mean value of $E$ corresponding to $\bar{t}$ or $R \gamma$, as a constant quantity for many electrolytes even if $t$ changes in a limited range. The fact that $\gamma_{ \pm} \min -\sqrt{m_{\min }}$ curves can be expressed using $\bar{t}$ [see Eq. (5)], will suggest that the constancy of $\bar{E}$ will be realized in real electrolytic solutions, although the potential energy between ions in real electrolytic solution will presumably be more complicated than $E$.

Further, we shall consider the meaning of the constancy of $\bar{E}$ in detail. From Eq. (11) we obtain at constant $e$ and $D$

$$
q /\left(b^{3 / 2} \alpha\right) \cong \text { constant }
$$

This will mean the distance of closest approach $\alpha$ is in relation to the quantity corresponding to the effective charge $q / b^{3 / 2}$ owing to the ionic interaction and the hydration effect. For a given type of electrolyte, we have from the definition of $b^{*}$,

$$
\frac{q}{b^{3 / 2} \alpha}=\frac{q}{\sqrt{(30 / \alpha)[h-(\nu / 2)]}}=\text { constant }
$$

so that

$$
(h-\text { constant }) \propto \alpha,
$$

that is, the distance of closest approach is proportional to the hydration number approximately.

In addition it is very interesting to compare $E=q e^{2} / b^{3 / 2} D_{\alpha}$ [Eq. (11)] with $E^{\prime}=\left|z_{1} z_{2}\right| e^{2} / D \alpha$, which is a potential energy between the two ions bearing $z_{1} e, z_{2} e$ charges at a distance $\alpha$ in a medium of dielectric constant $D$. Then from Table 1 we find the relation for all types

$$
E>E^{\prime}
$$

because $1>b>0$. This relation would be interpreted reasonably considering the decrease of the effective dielectric constant in the neighbourhood of ions due to the hydration or the saturation of water molecule by extremely strong electric field ( $\cong 10^{5}$ volt $/ \mathrm{cm}$ for a point charge $e$ ) of the ions ${ }^{5-9}$ ).

Finally, we shall consider the many-parameter equation for $\gamma_{ \pm}$. By increasing the number of parameters, it will naturally be expected to obtain a better agreement between the theory and the experiment. Here we shall consider Glueckauf's three-parameter equation for $\gamma_{ \pm}$based on the volume fractional statistics ${ }^{2), 10)}$,

* From the dimensional consideration, $b$ has been defined as follows: $30[h-(\nu / 2)]=(b \alpha)^{\mathbf{3}}$. See ref. 3 . 
that is, ${ }^{*}$

$$
\ln \gamma_{ \pm}=-\frac{A \sqrt{m}}{1+B \sqrt{m}}+\frac{\beta p(p+h-\nu)}{\nu(1+\beta p m i)}+\left(\frac{h-\nu}{\nu}\right) \ln (1+\beta p m)-\frac{h}{\nu} \ln (1-\beta h m)
$$

where $p$ is a ratio of the apparent molar volume of unhydrated electrolyte to the partial molar volume of free water. By the same procedure as in the preceding paper ${ }^{3)}$, we have

$$
R \gamma \equiv \frac{m_{1}}{m_{\min }}=\left(\frac{t_{v}^{1 / 2}-1}{t_{v}^{1 / 3}+\frac{4}{9} t_{v}^{-1 / 3}-\frac{4}{3}}\right)^{2}
$$

in a close approximation, where

$$
t_{v}=\frac{4 A B \nu}{\beta} /\left[(h+p)^{2}-2 p \nu\right]
$$

From Eqs. (2) and (14) we obtain

$$
\frac{t_{v}}{t}=\frac{\nu(2 h-\nu)}{(h+p-\nu)^{2}+\nu(2 h-\nu)}<1 \quad \text { for } h+p \neq \nu
$$

so that

$$
t_{v}<t
$$

Although we cannot easily give a simple meaning to $t_{v}$ as in the case of $t$, we recover Eq. (2) from (14) by putting $h+p=\nu^{* *}$. If we plot $R \gamma$ against $t_{v}$ according to Eq. (13), we may have a curve represented by a following linear relation

$$
R \gamma=5.2+0.0173 t_{v}
$$

and we may expect smaller value of $R \gamma$ in order to reproduce $\gamma_{ \pm \min }-\sqrt{m_{\min }}$ curve (See Fig. 5). In this case the original equation (12) can be written in the form

$$
\ln \gamma_{ \pm}=-\frac{A \sqrt{m}}{1+B \sqrt{m}}+\frac{\beta}{\nu}\left[(h+p)^{2}-2 p \nu\right] \mathrm{m}+\frac{\beta^{2}}{2}\left(h^{3}-3 h p^{2}+3 p^{2} \nu-2 p^{3}\right) m^{2}
$$

and this can also be reduced to the linearized Eq. ( $\left.4^{\prime}\right)$ putting $h+p=\nu$. Comparing the coefficient of $m$ in Eq. (4') with that in Eq. (17), one obtains

$$
\frac{\beta\left[(h+p)^{2}-2 p \nu\right]}{\nu}=\beta\left\{\frac{(h+p-\nu)^{2}}{\nu}+(2 h-\nu)\right\}>\beta(2 h-\nu) \quad \text { for } h+p \neq \nu
$$

Hence, the term containing $m$ in Eq. (17) become larger than that in Eq. ( $\left.4^{\prime}\right)$. Next, considering the magnitude of the coefficient of $m^{2}$ in Eq. (4'), we have

although for that in Eq. (17)

$$
\frac{3}{8}[\beta(2 h-\nu)]^{2}=\frac{3}{8}\left(\begin{array}{c}
\text { the second coefficient } \\
\beta(2 h-\nu)
\end{array}\right)^{2}
$$

$$
\frac{\beta^{2}}{2}\left(h^{3}-3 h p^{2}+3 p^{2} \nu-2 p^{3}\right)<\frac{3}{8}\left(\begin{array}{c}
\text { the second coefficient } \\
\frac{\beta}{\nu}\left[(h+p)^{2}-2 p \nu\right]
\end{array}\right)^{2}
$$

so that the increase in $\ln \gamma_{ \pm}$in Eq. (17) due to the third term becomes smaller than that in Eq. (4'). Accordingly the values of $\alpha$ will become smaller, and the better agreement between Eq. (17) and experiment will be expected. Only when the third term can be neglected, Eq. (17) can be reduced to one-parameter equation, that is

$$
\ln \gamma_{ \pm}=-\frac{A \sqrt{m}}{1+B \sqrt{m}}+\left(\frac{4 A B}{t_{v}}\right) m
$$

\section{Acknowledgment}

The author wishes to express his sincere thanks to professor M. Toda of Tokyo University of Education for his very kind encouragements and useful advices.

\section{(Received Mar. 31, 1961)}

* On the right of Eq. (12), the first term represents the Debye-Hückel term, the second the contribution from the difference in size of hydrated ion and solvent molecule. The other two terms indicate the contribution from the hydrated water and the difference of scale in the case of the volume fractional statistics.

** The conditinn $h \div p=\nu$, means the neglection of the size difference between the solute and solvent molecu'es. 


\section{Literature :}

1) M. Moriyama, Naturwissenschaften 43, 515 (1956); Z. physik. Chem. (Frankfurt) 27, 34 (1961).

2) E. Glueckauf, Trans. Faraday Soc. 51, 1,235 (1955); ibid. 53, 305 (1957). See also R. H. Stokes, R. A. Robinson, ibid. 53, 301 (1957).

3) M. Moriyama, Z. physik. Chem. (Frankfurt) 25, 310 (1960).

4) R. A. Robinson, R. H. Stokes, "Electrolyte Solution" p. 476 (1955) Butterworths Sci. Publ., London.

5) E. Hückel, Phys. Z. 26, 93 (1925).

6) J. D. Bernal, R. H. Fowler, J. Chem. Phys. 1, 515 (1933).

7) P. Debye, " Polare Molekeln" p. 128 (1929) Verlag von S. Hirzel, Leipzig.

8) J. B. Hasted, D. M. Ritson, C. H. Collie, J. Chem. Phys. 16, 1 (1948).

9) M. Moriyoma, J. Phys. Soc. Japan 8, 423 (1953).

10) M. Moriyama, T. Sugita, Denki-Kagaku 25, 617 (1957). 